\title{
Cost-benefit analysis of the intraoperative parathyroid hormone assay in primary hyperparathyroidism
}

\author{
Benedetta Badii, MD, ${ }^{1}$ Fabio Staderini, MD, ${ }^{1{ }^{\star}}$ Caterina Foppa, MD, ${ }^{1}$ Lorenzo Tofani, ${ }^{2}$ lleana Skalamera, MD, ${ }^{1}$ Giulia Fiorenza, MD, ${ }^{1}$ Eva Qirici, MD, ${ }^{1}$ \\ Fabio Cianchi, MD, ${ }^{1}$ Giuliano Perigli, MD ${ }^{1}$ \\ ${ }^{1}$ Unit of General and Endocrine Surgery, Center of Oncological and Minimally Invasive Surgery, Department of Surgery and Translational Medicine, University of Florence, Italy, \\ ${ }^{2}$ Department of Neurosciences, Psychology, Drug Research, and Child Health, University of Florence, Clinical Trials Coordinating Center, Istituto Toscano Tumori, Florence, Italy.
}

Accepted 22 July 2016

Published online 24 August 2016 in Wiley Online Library (wileyonlinelibrary.com). DOl 10.1002/hed.24567

\begin{abstract}
Background. The purpose of this study was to evaluate the usefulness of the routine intraoperative intact parathyroid hormone (IOPTH) assay, the role of unilateral and bilateral cervical exploration and of preoperative imaging, and to do a cost-benefit analysis in parathyroidectomy for primary hyperparathyroidism.

Methods. Two hundred sixty-four patients who underwent operations between January 2000 and March 2015 were retrospectively divided into 2 groups.

Results. Group A (IOPTH) was composed of 64 patients. Ultrasonography and technetium-99m-sestamibi (MIBI) identified the adenoma in 38 cases. Bilateral exploration was performed in 43 patients; of which 2 failures occurred. The IOPTH false-negative rate was $18.4 \%$. The
\end{abstract}

average cost was €1297.30. Group B (without IOPTH) was composed of 200 patients. Ultrasonography and MIBI identified the adenoma in 113 cases. Bilateral exploration was performed in 129 patients; of which 2 failures occurred. The average cost was $€ 618.75$.

Conclusion. The IOPTH assay should be used only in few selected cases because of its high cost. The experience of the team is essential to obtain a high cure rate. (c) 2016 Wiley Periodicals, Inc. Head Neck 39: 241-246, 2017

KEY WORDS: primary hyperparathyroidism, intraoperative parathyroid hormone (PTH) assay, minimally invasive parathyroid surgery, intraoperative parathyroid hormone (PTH) cost analysis

\section{INTRODUCTION}

Parathyroidectomy for primary hyperparathyroidism can be a challenging operation even when performed by an expert surgeon. Long-term cure rates of primary hyperparathyroidism at first operation are reported to be up to 95\% in referral centers. ${ }^{1}$ Despite this high percentage of success, the surgeon's goal is to achieve an efficacy as close as possible to $100 \%$, because reinterventions are difficult and are associated with high complication rates. ${ }^{2-4}$

Since the introduction of the intraoperative parathyroid hormone (IOPTH) assay ${ }^{5,6}$ and the improved accuracy of preoperative localization imaging, such as technetium99m-sestamibi (MIBI) and ultrasonography, parathyroidectomy with bilateral cervical exploration has been progressively reduced in favor of focused parathyroidectomy.

An accurate preoperative localization of the affected gland is possible with MIBI and ultrasonography that, if combined, reach a sensitivity and specificity of up to $96 \%$ and $83 \%,{ }^{7}$ respectively. IOPTH plays a pivotal role in confirming the removal of all hyperfunctioning tissue. Nevertheless, the main concerns regarding IOPTH are

${ }^{*}$ Corresponding author: S. Fabio, Unit of General and Endocrine Surgery, Center of Oncological and Minimally Invasive Surgery, Department of Surgery and Translational Medicine, University of Florence, Florence, Italy.

E-mail: staderini.fabio@gmail.com longer operative time, high cost, and a nonnegligible rate of false-positive and false-negative results, especially for patients with multiglandular pathology. ${ }^{1}$ For these reasons, IOPTH is not always widely used. The high costs of rapid IOPTH lead some centers to use it in the central laboratory, but this approach, which allows a wider use of IOPTH, is even more time-consuming and expensive.

In the last 15 years, our surgical management of primary hyperparathyroidism has changed because of a progressive lack of financial resources; the improvement of preoperative imaging and the increasing experience of the surgical team have allowed us to use IOPTH only in a few selected cases.

The purpose of this retrospective study was to evaluate the usefulness of IOPTH and to do a cost-benefit analysis of it. We also evaluated the role of unilateral and bilateral cervical exploration and of preoperative imaging.

\section{PATIENTS AND METHODS}

From January 2000 to March 2015, 264 patients underwent parathyroidectomy for primary hyperparathyroidism at the General and Endocrine Surgery Unit of Florence University Hospital, Florence, Italy.

Consistently with the purpose of this study, in order to better analyze the role of the IOPTH assay, patients were retrospectively divided into 2 groups on the basis of parathyroid hormone (PTH) measurement (data from General 
and Endocrine Surgery Unit database) that was performed after the "intraoperative intact parathyroid hormone [iPTH] assay protocol," previously described by Barczyński et $\mathrm{al}^{1}$ and always evaluated according to Miami criteria. ${ }^{8}$

Patient groups were as follows: group A consisted of 64 patients with use of the rapid IOPTH assay ("IOPTH group"); group B consisted of 200 patients without the IOPTH assay who were further divided in two subgroups: "B ${ }_{1}$ group" was composed of 77 patients with the delayed laboratory PTH assay sampled during the surgical intervention but with results available on postoperative day 1; and the "B $\mathrm{B}_{2}$ group" consisted of 123 patients with no IOPTH assay at all.

The $\mathrm{B}_{1}$ group was considered our control group because the results of PTH intraoperative sampling could not change the surgeon's strategy during the operation and it was used to better understand if IOPTH would be helpful in guiding the surgeon's intraoperative decisions. Blood samples for the delayed PTH assay were taken according to "intraoperative iPTH assay protocol" and Miami criteria timing but sent to the central laboratory at the end of surgery.

Only patients with primary hyperparathyroidism were included in our study. Primary hyperparathyroidism was defined as increased PTH levels in a hyper/normocalcemic patient without vitamin $\mathrm{D}$ deficiency and with a normal renal function. Exclusion criteria were preoperative diagnosis of multiglandular pathology because of hyperplasia and multiple endocrine neoplasias.

Demographics, patients' medical history, clinical presentation (asymptomatic or symptomatic), association with thyroid disease, biochemical values, localization imaging, surgical procedures, histopathological results, complications, and outcomes were recorded and analyzed.

Patients with primary hyperparathyroidism and no signs or symptoms of primary hyperparathyroidism were defined as asymptomatic. Patients with signs or symptoms of primary hyperparathyroidism were defined as symptomatic.

Biochemical diagnosis was based on serum calcium, phosphorus, PTH, urinary phosphorus, and calcium. Hypercalcemia was defined as serum calcium $>10.4 \mathrm{mg}$ / dL $(8.5-10.1 \mathrm{mg} / \mathrm{dL})$. Hyper-PTH was defined as serum PTH $>5.8 \mathrm{pmol} / \mathrm{L}(1.3-7.6 \mathrm{pmol} / \mathrm{L})$.

Preoperative localization was based on MIBI, ultrasonography, or both. When both were available, their concordance in locating the adenoma was recorded (Table 1).

The surgical approach was either a minimally invasive video-assisted parathyroidectomy (MIVAP), incision $<25$ $\mathrm{mm}$ or mini-incision parathyroidectomy, incision $<35 \mathrm{~mm}$; or conventional parathyroidectomy, incision $>35 \mathrm{~mm}$.

Bilateral exploration was deemed mandatory when there was no concordance of preoperative imaging in locating the adenoma, when the intraoperative finding did not correspond to preoperative imaging, or when the ipsilateral gland to the adenoma was hyperplastic or not in the usual site, and in cases of associated thyroidectomy. In all other cases, a monolateral exploration with the identification of the ipsilateral gland was performed.

Serum calcium and PTH were assayed in all patients on postoperative days 1 and 7 to confirm the cure or the persistence of the disease. Surgical failure was confirmed
TABLE 1. Patient demographics, preoperative, and postoperative data.

\begin{tabular}{|c|c|c|}
\hline & $\begin{array}{c}\text { Group } A=64 \\
\text { patients (\%) }\end{array}$ & $\begin{array}{c}\text { Group } B=200 \\
\text { patients (\%) }\end{array}$ \\
\hline \multicolumn{3}{|l|}{ Demographics } \\
\hline $\mathrm{M}: \mathrm{F}$ & $16: 48$ & $46: 154$ \\
\hline Mean age (range), y & $61(33-83)$ & $60(20-88)$ \\
\hline Symptomatic & $41(64)$ & $84(42)$ \\
\hline Asymptomatic & $24(36)$ & $116(58)$ \\
\hline Preoperative hypercalcemia & $50(78)$ & $155(77.5)$ \\
\hline \multicolumn{3}{|l|}{ Preoperative imaging } \\
\hline Ultrasonography only & $3(5)$ & $36(18)$ \\
\hline MIBI only & $2(3)$ & $5(2.5)$ \\
\hline Ultrasonography + MIBI & $59(92)$ & $159(79.5)$ \\
\hline \multicolumn{3}{|l|}{$\begin{array}{l}\text { MIBI + ultrasonography } \\
\text { concordance }\end{array}$} \\
\hline Yes:no & $44: 15$ & $121: 38$ \\
\hline $\mathrm{N} / \mathrm{A}$ & 5 & 41 \\
\hline \multicolumn{3}{|l|}{ Surgical procedure } \\
\hline Conventional & $43(67)$ & $120(60)$ \\
\hline $\begin{array}{l}\text { Mini-incision } \\
\text { parathyroidectomy }\end{array}$ & $13(20)$ & $80(40)$ \\
\hline MIVAP & $8(13)$ & $0(0)$ \\
\hline \multicolumn{3}{|l|}{ Exploration } \\
\hline Monolateral & $21(33)$ & $71(35.5)$ \\
\hline Bilateral & $43(67)$ & $129(64.5)$ \\
\hline \multicolumn{3}{|l|}{ Associated thyroid surgery } \\
\hline Yes:no & $29: 40$ & $102: 98$ \\
\hline Goiter & 25 & 89 \\
\hline Carcinoma & 4 & 13 \\
\hline \multicolumn{3}{|l|}{ Outcomes } \\
\hline Success & $62(96.8)$ & $198(99)$ \\
\hline Failure & $2(3.2)$ & $2(1)$ \\
\hline \multicolumn{3}{|l|}{ Reinterventions } \\
\hline Success & 1 & 2 \\
\hline Failure & 0 & 0 \\
\hline \multicolumn{3}{|l|}{ Pathology results } \\
\hline Adenoma & 64 & 200 \\
\hline Single & $58(90.6)$ & $193(96.5)$ \\
\hline Double & $6(9.4)$ & $7(3.5)$ \\
\hline Complications & $4(6.25)$ & $13(6.5)$ \\
\hline
\end{tabular}

Abbreviations: MIBI, technetium-99m-sestamibi; N/A, not applicable; MIVAP, minimally invasive video-assisted parathyroidectomy.

by persistent hypercalcemia with inappropriately elevated PTH.

The cost-benefit analysis of groups A and B was performed by considering group $\mathrm{B}$ as divided into the 2 subcategories $B_{1}$ and $B_{2}$ groups. Average costs for a single patient were calculated by considering the cost of the rapid IOPTH assay ( $€ 170$ for 5 measurements in our institution), of the delayed PTH assay (€125 for 5 measurements in our institution), and of the operating room ( $€ 15$ per minute in our institution). Costs of reoperations for surgical failures were also recorded and added to the total cost of the related group (Table 2).

\section{Statistics}

To evaluate the association between dichotomous variables, the Fisher's exact test was used. To assess the normality of the distribution of continuous variables, the Shapiro-Wilke test was used.

The Kruskall-Wallis test and the Dwass, Steel, Critchlow Fligner test for multiple comparisons were used to 
TABLE 2. Cost-benefit analysis (Euros).

\begin{tabular}{lccc}
\hline & Group A $(n=64)$ & Group B $(n=77)$ & Group B $(n=123)$ \\
\hline Mean operative time (min) & $73.84(30-210)$ & $40(20-105)$ & $40(12-100)$ \\
OR costs* (15 Euro/min) & 1107.30 & 600 & 600 \\
IOPTH costs ${ }^{*}$ (170 Euro 5 assays) & 170 & - & - \\
Laboratory assay costs (25 Euro 5 assays) & 1277.30 & 725 & - \\
Totals & 1277.30 & - & 600 \\
Reintervention costs & $1170.43(1070)$ & $615.32(537.5)$ & 1200 \\
Average (median) cost per patient & & $491.28(450)$ \\
\hline
\end{tabular}

Abbreviation: OR, operating room

*Average costs calculated for a single patient.

evaluate the association between categorical and continuous variables.

The proportion of agreement cases were used to describe the concordance between diagnostic techniques and histopathology.

The significance level was set at $5 \%$.

\section{RESULTS}

\section{Group A}

In group $\mathrm{A}$, there were 64 patients, 48 women $(75 \%)$ and 16 men (25\%), with a mean age of 61 years (range, 33-83 years). Forty-one patients (64\%) had signs and symptoms of primary hyperparathyroidism that most frequently were musculoskeletal pain and nephrolithiasis. Fifty patients $(78 \%)$ had preoperative hypercalcemia, whereas the others were normocalcemic because of medical treatment.

Both ultrasonography and MIBI were performed in 59 patients and were concordant in 44 cases $(74.5 \%)$. Ultrasonography alone was performed in 3 cases, whereas only MIBI was performed in 2 cases. Of the 44 cases in which ultrasonography and MIBI were concordant, they were able to correctly identify the adenomas in 38 (86.3\%). Among the other 6 patients, ultrasonography and MIBI showed a single adenoma instead of a double adenoma (ipsilateral) in 3 patients, they were not able to localize any adenoma in 2 patients, and they were not able to localize a second contralateral adenoma in 1 patient.

Conventional parathyroidectomy was performed in 43 patients $(67 \%)$, mini-incision parathyroidectomy in 13 patients $(20.3 \%)$, and MIVAP in 8 patients $(12.5 \%)$. Monolateral exploration was performed in 21 patients (33\%) and bilateral exploration in 43 patients $(67 \%)$. Twenty-nine patients $(45.3 \%)$ underwent an obliged bilateral exploration because of associated thyroid surgery (4 carcinomas and 25 goiters). The other 14 patients $(21.8 \%)$ had bilateral explorations that were performed because IOPTH did not decrease: 2 (3\%) were true-negatives, suggesting the failure of the intervention, but $12(18.4 \%)$ were false-negatives and IOPTH compelled us to perform unnecessary bilateral explorations.

Pathology was consistent with single adenoma in 58 patients $(90.6 \%)$ and with double adenoma in 6 patients (9.4\%).

Four patients $(6.25 \%)$ had complications, 2 had transient recurrent nerve palsy that resolved within 1 month and 2 had transient hypocalcemia treated with oral calcium and vitamin D that resolved within 2 weeks. All these patients had undergone bilateral explorations.

At 1 month of follow-up, 62 patients $(96.8 \%)$ reached normal values of calcium and PTH. Two patients had persistent disease: 1 underwent a successful reintervention and the other did not undergo reoperation (Table 1).

The mean total operative time in those cases without associated thyroidectomy was 73.84 minutes (range, 30150 minutes). The average cost of a parathyroidectomy with IOPTH was $€ 1297.30$ (operating room + IOPTH + reintervention costs; Table 2).

\section{Group B}

In group B, there were 200 patients, 154 women (77\%) and 46 men $(23 \%)$, with a mean age of 60 years (range, 20-88 years). Eighty-four patients (42\%) had signs and symptoms of hyperparathyroidism that most frequently were musculoskeletal pain and nephrolithiasis. One hundred fifty-five patients $(77.5 \%)$ had preoperative hypercalcemia, whereas the others were normocalcemic because of medical treatment.

Both ultrasonography and MIBI were performed in 159 patients and were concordant in 121 patients (76\%). Only ultrasonography was available in 36 patients, MIBI alone was performed in 5 patients.

Of the 121 patients in whom ultrasonography and MIBI were concordant, they correctly identified the site of the adenoma in 113 patients $(93.3 \%)$. In 5 patients $(6.7 \%)$, neither of them identified any adenomas that instead were found at surgery, because of bilateral cervical exploration. In this case, indications for surgery were hypercalcemia and hyper-PTH associated with sign and symptoms of primary hyperparathyroidism. In 9 cases $(7.4 \%)$, both types of imaging were side concordant but one of them described another contralateral adenoma not found at surgery. In 3 cases $(2.4 \%)$, preoperative imaging was concordant but surgery revealed that the adenoma was on the other side.

Conventional parathyroidectomy was performed in 120 patients $(60 \%), 80(40 \%)$ of whom underwent miniincision parathyroidectomy and none underwent MIVAP. Monolateral exploration was performed in 71 cases $(35.5 \%)$ and bilateral exploration in 129 cases $(64.5 \%)$. One hundred two patients $(51 \%)$ in whom a bilateral exploration was performed underwent associated thyroid surgery, 13 for thyroid carcinomas and 89 for goiters. Pathology was consistent with a single adenoma in 193 patients $(96.5 \%)$ and with a double adenoma in 7 (3.5\%). 
TABLE 3. Statistical analysis.

\begin{tabular}{ll}
\hline & $p$ value \\
\hline Complications & \\
Side concordance of imaging & .4 \\
Monolateral/bilateral exploration & .08 \\
Thyroidectomy & .6 \\
OR time & .01 \\
Failures & .2 \\
Group A vs group B & .3 \\
Monolateral vs bilateral exploration & .3 \\
Side concordance of imaging & $<.0001$ \\
Costs & $<.0001$ \\
IOPTH vs delayed & .2 \\
IOPTH vs no PTH & \\
Delayed vs no PTH & \\
\hline
\end{tabular}

Abbreviations: OR, operating room; IOPTH, intraoperative intact parathyroid hormone; PTH, parathyroid hormone.

Figures in boldface indicate statistical significance.

Thirteen patients $(7 \%)$ had complications, 1 had transient hypocalcemia treated with oral calcium and vitamin $\mathrm{D}$ that resolved within 2 weeks, 2 patients had hematomas conservatively treated, and 10 patients had transient recurrent nerve palsies that resolved within 1 month. Of these, 11 patients had been operated with bilateral and 2 with monolateral exploration. Two failures occurred in the $\mathrm{B}_{2}$ group; these 2 patients underwent successful reoperations.

All 77 patients in Group $B_{1}$ were considered treated because the delayed PTH assay decreased, in accord with Miami criteria (Table 1).

The mean operating time in those cases without associated thyroidectomy was 40 minutes. The average cost of a parathyroidectomy with delayed PTH was €725 (operating room + delayed PTH costs), whereas the average cost of a parathyroidectomy without IOPTH was $€ 618.75$ (operating room + reinterventions costs; Table 2).

When the results of both groups were combined, MIBI and ultrasonography together showed specificity in identifying the side of the adenoma of $80 \%$ and $69.92 \%$, respectively; when combined, they had a specificity of $79.82 \%$. Even though bilateral exploration was associated with more complications in both groups, the difference was not statistically significant (Table 3 ). There was no significant association between preoperative imaging concordance in locating the adenomas and complications (Table 3).

Associated thyroid surgery was not significantly associated with complications, although operative time was (Table 3).

No difference in failure rate was observed in the 2 groups. Both monolateral/bilateral explorations and preoperative/intraoperative side concordances were not associated with failures (Table 3).

The cost-benefit analysis was performed by splitting group B into 2 different subgroups: $\mathrm{B}_{1}$ ("delayed PTH group") and $\mathrm{B}_{2}$ ("no PTH group"). The costs were significantly different between group A "IOPTH group" and group $\mathrm{B}_{1}$ and between group $\mathrm{A}$ and group $\mathrm{B}_{2}$ (Table 3 ).

\section{DISCUSSION}

Preoperative localization imaging, such as MIBI and ultrasonography, has now achieved high accuracy. Nevertheless, their combination reaches even higher sensitivity in locating parathyroid adenomas, results are heterogeneous in the literature ranging from $80 \%$ to $98 \% .^{9}$ These good results have allowed a large diffusion of the unilateral approach and minimally invasive parathyroidectomy. ${ }^{10}$ Unilateral and focused surgery is, in fact, feasible in patients with preoperative imaging consistent with uniglandular disease ${ }^{11}(85 \%$ of patients with primary hyperparathyroidism), but the main drawback of minimalaccess surgery is that it does not allow the surgeon to see all 4 parathyroid glands. For this reason, many studies have encouraged the use of the IOPTH assay ${ }^{5,6}$ because, according to "intraoperative iPTH assay protocol" and the Miami criteria, ${ }^{1,8}$ a PTH decrease greater than $50 \%$ of preoperative values 10 minutes after the removal of the hyperfunctioning gland corresponds to a cure. Authors in favor of the IOPTH assert that it can alert the surgeon to the presence of multiglandular disease that is diagnosed in up to $10 \%$ of patients with primary hyperparathyroidism. ${ }^{12}$ At the same time, other authors have demonstrated its nonnegligible rate of false-positives and falsenegatives, especially in patients with multiglandular pathology. ${ }^{1,13}$ In 2004, the Mayo Clinic reported that patients with primary hyperparathyroidism and unequivocally positive preoperative localization can be successfully managed by focused unilateral cervical exploration without IOPTH measurements. ${ }^{14}$ From these reports, several studies have not sustained the routine assay of IOPTH. $^{15,16}$

In our case series, patients underwent parathyroidectomy over a period of time (2000-2015) that allowed us to assess the development of diagnostic techniques and surgical treatments.

We divided patients into 2 groups to better analyze the costs and benefits of IOPTH, and to evaluate the role of unilateral and bilateral cervical exploration and of preoperative imaging.

We registered 2 (3\%) lacks of success in group A, and $2(1 \%)$ in group B.

With regard to group A, 1 patient was operated for a right inferior adenoma identified by ultrasonography but not by MIBI. Because IOPTH did not decrease after the removal of the first adenoma, bilateral exploration was performed but the surgeon failed to find another abnormal gland. The patient underwent another ultrasonography and an MIBI that showed another adenoma on the left side that was successfully removed at reoperation. The other failure was related to a missing adenoma preoperatively localized by ultrasonography and MIBI. Persistently high IOPTH levels led the surgeon to perform a bilateral exploration, but the adenoma was not found. Both an ultrasonography and an MIBI were repeated and they confirmed the adenoma. This patient was not reoperated because of severe cardiac problems that developed 1 month after surgery.

As confirmed by our experience, IOPTH influences the surgical management of primary hyperparathyroidism but it is still unable to guarantee a $100 \%$ cure rate because the surgeon's experience still plays a pivotal role in finding the adenomas. Furthermore, it is important to focus on the $18.4 \%$ of false-negatives in which a decrease of less than $50 \%$ of IOPTH compelled the surgeon to carry out a useless bilateral exploration with important 
implications for operating time and in terms of a more invasive surgical technique with a higher risk of complications.

We registered 2 failures in group B. One patient had concordant preoperative imaging for a right-sided parathyroid adenoma but a bilateral cervical exploration revealed a left not a right adenoma. Because the postoperative PTH did not decrease, this patient underwent another MIBI that confirmed the right-sided adenoma again. The patient was reoperated and the right adenoma was found and removed. In addition, the second patient had concordant preoperative imaging and the adenoma was found and removed without performing a bilateral cervical exploration. Because the late postoperative PTH did not decrease, the patient repeated ultrasonography and MIBI that revealed a second adenoma on the other side, which was previously undiagnosed. The patient underwent a successful reoperation. In this last case, IOPTH may have potentially suggested the second adenoma and, thus, increased the cure rate.

Many authors report about the importance of preoperative imaging in parathyroid surgery. ${ }^{9-11}$ Our experience confirms the importance of ultrasonography and MIBI in planning on which side to perform the cervical exploration. When combined, they reach a specificity of $80 \%$, a result that agrees with data reported in the literature even if it could be higher considering only recent series. Specificity could have been affected by the learning curve of the operators who performed imaging studies and by the improved quality of imaging tools over the 15 years of the study. Preoperative side concordance with an intraoperative finding of the adenoma is associated with a high cure rate ( $97.3 \%$ in our experience).

Unilateral neck exploration is associated with fewer recurrent nerve complications, no risk of hypoparathyroidism, and it is easier to perform. Even though bilateral exploration is associated with more complications, the difference is not statistically significant, and bilateral exploration provides a more accurate evaluation in cases of associated thyroid surgery, discordant or negative preoperative imaging, and suspicion of multiglandular pathology. We also have to consider that, in $20 \%$ of cases, MIBI and ultrasonography were not able to localize the adenoma on the correct side, and, in these cases, bilateral exploration is of utmost importance to avoid failure. We need to consider that a systematic bilateral exploration could be associated with a higher success rate and comparable operating time, postoperative pain, length of stay, and aesthetic results, as Norman et $\mathrm{al}^{17}$ previously reported.

A hypothetic approach could begin with a unilateral neck exploration on the side of the suspected adenoma. After its removal, always explore the ipsilateral gland: a normotrophic, normotopic gland could suggest uniglandular disease, whereas a normotopic, hypertrophic gland or an ectopic gland could suggest multiglandular disease and then bilateral exploration should be mandatory even because, in our experience, bilateral exploration was not significantly associated with complications. This approach is not validated yet, as further studies are needed to confirm its efficacy.
Surgeons must be aware that operative time increases when a focused approach is performed and the adenoma is not found on the side suggested by preoperative imaging. In some of these cases, the adenoma is on the other side because of the failure of preoperative imaging and the meticulous monolateral research can increase the operative time and the risk of complications. In these cases, bilateral exploration should be considered.

Our cost-benefit analysis shows a substantial difference between our 2 groups of patients. This is mostly because of longer operative times in group A. Comparing groups $\mathrm{B}_{1}$ and $\mathrm{A}$, the cost saving per patient amounts to $€ 572.30$, and it increases to $€ 678.55$ per patient when groups $B_{2}$ and $\mathrm{A}$ are compared.

We are convinced that the presence of an expert surgical team dedicated to the treatment of this pathology is essential, as affirmed by Norman et $\mathrm{al}^{17}$ and earlier by the NIH conference ${ }^{18}$ : "The greatest challenge in parathyroid localization is to localize a good parathyroid surgeon."

The limitations of this study are due to the small number of failures, meaning the statistical analysis of failures is not completely reliable and that patients in group B underwent operations when the surgeon's and the team's experience reached the highest level. Another bias of this study could be the learning curve of operators and the improvement of technologies during the last 15 years analyzed.

There is no doubt, however, about the considerable economic benefits of not using IOPTH except when absolutely necessary, ${ }^{19}$ as our study demonstrates. There are also other relevant benefits, including shorter operating time, which may have favorable effects on the patient and results in fewer complications, shorter anesthesia, and length of hospital stay. Shorter operating time can also increase the number of patients operated per day and, thus, decrease the waiting list. Our experience confirms that, in high volume centers with a dedicated and welltrained surgical team, the routine use of IOPTH is not mandatory. We reserve its use for reoperations for parathyroid pathology and after any re-cervicotomy.

It is otherwise important to confirm the fundamental role of IOPTH in selected cases and most importantly in the training of endocrine surgeons.

\section{CONCLUSIONS}

Our single-center analysis of patients treated for primary hyperparathyroidism suggests that the intraoperative PTH assay can no longer be used routinely but only in selected cases. This approach can decrease costs and obtain other benefits to the patient, such as shorter operation time, fewer complications, and reduced hospital stay. Nevertheless, the experience of the surgical team is essential to obtain a high cure rate.

\section{REFERENCES}

1. Barczyński M, Gołkowski F, Nawrot I. The current status of intraoperative iPTH assay in surgery for primary hyperparathyroidism. Gland Surg 2015; 4:36-43.

2. Beazley RM, Costa J, Ketcham AS. Reoperative parathyroid surgery. Am J Surg 1975;130:427-429.

3. Jaskowiak N, Norton J, Alexander HR, et al. A prospective trial evaluating a standard approach to reoperation for missed parathyroid adenoma. Ann Surg 1996;224:308-320; discussion 320-321. 
4. Cheung PS, Borgstrom A, Thompson NW. Strategy in reoperative surgery for hyperparathyroidism. Arch Surg 1989;124:676-680.

5. Chen H, Pruhs Z, Starling JR, Mack E. Intraoperative parathyroid hormone testing improves cure rates in patients undergoing minimally invasive parathyroidectomy. Surgery 2005;138:583-587; discussion 587-590.

6. Irvin GL III, Prudhomme DL, Deriso GT, Sfakianakis G, Chandarlapaty SK. A new approach to parathyroidectomy. Ann Surg 1994;219:574-579; discussion 579-581.

7. De Feo ML, Colagrande S, Biagini C, et al. Parathyroid glands: combination of $(99 \mathrm{~m})$ Tc MIBI scintigraphy and US for demonstration of parathyroid glands and nodules. Radiology 2000;214:393-402.

8. Carneiro DM, Solorzano CC, Nader MC, Ramirez M, Irvin GL III. Comparison of intraoperative iPTH essay (QPTH) criteria in guiding parathyroidectomy: which criterion is the most accurate? Surgery 2003;134:973979; discussion 979-981.

9. Haber RS, Kim CK, Inabnet WB. Ultrasonography for preoperative localization of enlarged parathyroid glands in primary hyperthyroidism: comparison with $(99 \mathrm{~m})$ technetium sestamibi scintigraphy. Clin Endocrinol (Oxf) 2002;57:241-249.

10. Sackett WR, Barraclough B, Reeve TS, Delbridge LW. Worldwide trends in the surgical treatment of primary hyperparathyroidism in the era of minimally invasive parathyroidectomy. Arch Surg 2002;137:1055-1059.

11. Ghandur-Mnaymneh L, Kimura N. The parathyroid adenoma. A histopathologic definition with a study of 172 cases of primary hyperparathyroidism. Am J Pathol 1984;115:70-83.
12. Clerici T, Brandle M, Lange J, Doherty GM, Gauger PG. Impact of intraoperative parathyroid hormone monitoring on the prediction of multiglandular parathyroid disease. World J Surg 2004;28:187-192.

13. Gauger PG, Agarwal G, England BG, et al. Intraoperative parathyroid hormone monitoring fails to detect double parathyroid adenomas: a 2-institution experience. Surgery 2001;130:1005-1010.

14. Jacobson SR, van Heerden JA, Farley DR, et al. Focused cervical exploration for primary hyperparathyroidism without intraoperative parathyroid hormone monitoring or use of the gamma probe. World J Surg 2004;28:1127-1131.

15. Suliburk JW, Sywak MS, Sidhu SB, Delbridge LW. 1000 minimally invasive parathyroidectomies without intra-operative parathyroid hormone measurement: lessons learned. ANZ J Surg 2011;81:362-365.

16. Gawande AA, Monchik JM, Abbruzzese TA, Iannuccilli JD, Ibrahim SI Moore FD Jr. Reassessment of parathyroid hormone monitoring during parathyroidectomy for primary hyperparathyroidism after 2 preoperative localization studies. Arch Surg 2006;141:381-384; discussion 384.

17. Norman J, Lopez J, Politz D. Abandoning unilateral parathyroidectomy: why we reversed our position after 15,000 parathyroid operations. J Am Coll Surg 2012;214:260-269.

18. [No authors listed]. NIH conference. Diagnosis and management of asymptomatic primary hyperparathyroidism: consensus development conference statement. Ann Intern Med 1991;114:593-597.

19. Morris LF, Zanocco K, Ituarte PH, et al. The value of intraoperative parathyroid hormone monitoring in localized primary hyperparathyroidism: a cost analysis. 2010;17:679-685. 